\title{
Design of Automatic Carrier Landing System Based on Dynamic Inverse and PID Control
}

\author{
Hengzhi Ding ${ }^{1, \text { a }}$ \\ ${ }^{1}$ School of Beihang University, Beijing 100191, China. \\ aDshinji137@163.com.
}

Keywords: carrier air wake, inverse dynamic, PID control

\begin{abstract}
At last twenty seconds of carrier landing process, the carrier-based aircraft will encounter with strong carrier air wake which will easily cause a deviation in the aircraft's path, at the same time, a carrier also has a six-degree-of-freedom motion which depends on carrier's property and sea state [6]. Without an efficient control in the aircraft, it will easily result a fatal crash. In this paper, the author use the theory of nonlinear inverse dynamics combined with PID control to design an automatic carrier landing system which can efficiently resist carrier air wake and can limit lateral position error and vertical height error in $1 \mathrm{~m}$ and longitudinal position error in $5 \mathrm{~m}$ on sea state level 4 in the simulation of Simulink.
\end{abstract}

\section{Introduction}

The last twenty seconds of landing process is faced with great risk mainly because of strong carrier air wake. According to statistics, American carrier landing had an accident rate of $0.031 \%$ in the day time and even $0.1 \%$ in the night in 1964 which greatly exceeded that of land-based landing [8]. Additionally, considering that the longitudinal axis of carrier and that of landing deck has an angle of about 9 degrees, the aircraft should have a transverse velocity to compensate the cross move of carrier, the sideslip angle $\beta$ is not equal to zero in this period, either. Through linearizing dynamical and kinematical equations of aircraft on the condition of little perturbation, we find that longitudinal and lateral variables have a comparatively strong coupling, which indicates separately using a longitudinal control loop and a lateral control loop to exert control is not an efficient way during last stage of carrier landing. Part of aircraft's dynamical and kinematical equations of aircraft can be written in forms of formula 1 , which is an express of a nonlinear system. When dealing with nonlinear system, dynamic inverse is a commonly used method. It can avoid complex parameters setting and gain adjustment. As long as we know the accurate mathematical model of a system, dynamic inverse can be applied to exert control [7, 10]. Knowing exactly about aircraft's dynamical and kinematical equations, dynamic inverse is a feasible way for flight control.

$$
\left\{\begin{array}{l}
\dot{x}=f(x)+g(x) u \\
y=H(x)
\end{array}\right.
$$

\section{Carrier landing safety standard}

There are in total four arresting cables installed on landing deck, and there is an ideal touch point located at the midpoint of second and third arresting cable. One of the standards of evaluating security and accuracy of carrier landing is landing position error. It has three components: lateral position error, vertical height error and longitudinal position error. Lateral position error is deviation of aircraft's longitudinal axis to deck's longitudinal axis when the aircraft lands on deck. Vertical height error is vertical difference between actual landing path and ideal landing path when aircraft flies right above ideal touch point. Longitudinal position error is horizontal distance between actual touch point and ideal touch point [1]. Its level classification standard is shown in table 1 [1]. 
Table 1 Carrier landing error classification (unit: meter)

\begin{tabular}{ccc}
\hline Error tyep & Ideal value & Allowable value \\
\hline Longitudinal position & $-6.1 \sim 6.1$ & $-12.2 \sim 12.2$ \\
Vertical height & $-0.76 \sim 1.52$ & $-1.52 \sim 3.05$ \\
Lateral position & $-1.52 \sim 1.52$ & $-3.05 \sim 3.05$ \\
\hline
\end{tabular}

It is ideal for an aircraft to touch a point whose landing error is in ideal range. If an aircraft lands at a point whose landing error is beyond ideal range but in allowable range, it can be acceptable but with less security. But if an aircraft is unfortune to land out of allowable range, it can lead serious conseuquence such as crasing carrier in advance or falling into the ocean [1]. It is therefore significant to make the probabitliy of landing at ideal area as large as possible.

\section{Modeling}

\subsection{Carrier air wake}

According to Military Standard $M I L-H D B K-1797 C$, there are four sorts of carrier air wake, they are free atmospheric turbulence component, cocktail component, periodic component and random component. [2,3] Cocktail and periodic components contribute mainly to carrier air wake, these two components are functions of wind over the deck, aircraft flying altitude, longitudinal distance between aircraft and ideal touch point as well as carrier motion.

\subsection{Coordinate system}

In order to construct a model for aircraft and provide an available condition for simulation, several coordinate systems should be introduced.

1. Body coordinate system $o_{b} x_{b} y_{b} z_{b}$ : Origin is centroid of an aircraft, $o_{b} x_{b}$ axis is parallel with aircraft's longitudinal axis and points to nose, $o_{b} z_{b}$ axis is in the plane of symmetry and perpendicular to $o_{b} x_{b}$ axis and points downwards, $o_{b} y_{b}$ axis is perpendicular to plane of $x_{b} o_{b} z_{b}$ and points to right of plane. They are in line with right-hand screw rule [4]. Six-degree-of-freedom dynamical equations are built in this coordinate system.

2. Wind coordinate system $o_{w} x_{w} y_{w} z_{w}$ : Origin is centroid of an aircraft, $o_{w} x_{w}$ axis is parallel with flying velocity vector, $o_{w} z_{w}$ axis is in the plane of symmetry and perpendicular to $o_{w} x_{w}$ axis and points downwards, $o_{w} y_{w}$ axis is perpendicular to plane of $x_{w} o_{w} z_{w}$ and points to right of aircraft. They are in line with right-hand screw rule [4]. Aerodynamic force vector on aircraft is initially decomposed in this axis, because dynamical equations are built in body axis, we need to convert aerodynamic force in wind coordinate system to that in body coordinate system.

3. Deck coordinate system $o_{d} x_{d} y_{d} z_{d}$ : Origin is ideal touch point, $o_{d} y_{d}$ axis is parallel with the longitudinal axis of deck, $o_{d} x_{d}$ axis is on the plane of deck and perpendicular to $o_{d} y_{d}$, pointing to right of the carrier, $o_{d} z_{d}$ axis is perpendicular to the plane of deck and points upwards. Because in simulation, carrier is going at a constant speed, and simulation time is short, this coordinate system is inertial system. We mainly focus on the location of aircraft in this coordinate system to judge if it can resist carrier air wake and land on the deck safely.

\subsection{Aircraft mathematical model}

In body-axis, establish six-degree-of-freedom dynamical equations as follows [4]. 


$$
\left\{\begin{array}{l}
\dot{u}=r v-q w+\frac{F_{x}}{m} \\
\dot{v}=p w-r u+\frac{F_{y}}{m} \\
\dot{w}=q u-p v+\frac{F_{z}}{m} \\
\dot{p}=\frac{I_{z} M_{x}-I_{z}\left(I_{z}-I_{y}\right) q r+I_{z} I_{x z} p q+I_{x z} M_{z}-I_{x z}\left(I_{y}-I_{x}\right) p q-I_{x z}^{2} q r}{I_{x} I_{z}-I_{x z}^{2}} \\
\dot{q}=\frac{M_{y}-\left(I_{x}-I_{z}\right) p r-I_{x z}\left(p^{2}-r^{2}\right)}{I_{y}} \\
\dot{r}=\frac{I_{x} M_{z}-I_{x}\left(I_{y}-I_{x}\right) p q-I_{x} I_{x z} q r+I_{x z} M_{x}-I_{x z}\left(I_{z}-I_{y}\right) q r+I_{x z}^{2} p q}{I_{x} I_{z}-I_{x z}^{2}}
\end{array}\right.
$$

In formula $2, F_{x}, F_{y}, F_{z}$ are resultant force on aircraft decomposed in body coordinate system, $M_{x}, M_{y}, M_{z}$ are resultant moment on aircraft decomposed in body coordinate system. $u, v, w$ are flying velocity $\mathrm{V}$ decomposed in body coordinate system. $p, q, r$ are rotate angular rate of aircraft decomposed in body coordinate system. In order to be more convenient for following control, replace $u, v, w$ with flying velocity $V$, attack angle $\alpha$ and sideslip angle $\beta$, based on formula 3 .

$$
\left\{\begin{array}{l}
\dot{V}=\frac{u \dot{u}+v \dot{v}+w \dot{w}}{V} \\
\dot{\alpha}=\frac{u \dot{w}-w \dot{u}}{u^{2}+w^{2}} \\
\dot{\beta}=\frac{\dot{v} V-v \dot{V}}{V^{2} \cos \beta} \\
u=V \cos \alpha \cos \beta \\
v=V \sin \beta \\
w=V \cos \alpha \sin \beta
\end{array}\right.
$$

Besides, establish six kinematical equations as formula 4 [4].

$$
\left\{\begin{array}{l}
\dot{\phi}=p+\tan \theta(q \sin \phi+r \cos \phi) \\
\dot{\theta}=q \cos \phi-r \sin \phi \\
\dot{\psi}=\frac{1}{\cos \theta}(q \sin \phi+r \cos \phi) \\
\dot{P}_{x}=u(\sin \psi \cos \theta+\sin \phi \cos \psi \sin \theta)+v \cos \psi \cos \phi+w(\sin \psi \sin \theta-\sin \phi \cos \psi \cos \theta) \\
\dot{P}_{y}=u(\cos \psi \cos \theta-\sin \psi \sin \phi \sin \theta)-v \sin \psi \cos \phi+w(\cos \psi \sin \theta+\sin \psi \sin \phi \cos \theta) \\
\dot{H}=u \cos \phi \sin \theta-v \sin \phi-w \cos \phi \cos \theta
\end{array}\right.
$$

In formula $4, \Phi, \theta, \psi$ stand for roll, pitch and yaw angle of aircraft. $P_{x}, P_{y}, H$ stand for aircraft's location in deck coordinate system. Because in perfect condition, value of $P_{x}$ should be zero, in following content, state variable $P_{x}$ will be regarded as lateral deviation. In formula 2-4, there are twenty state variables in total, they are $\left[\mathrm{V}, \alpha, \beta, \Phi, \theta, \psi, \mathrm{p}, \mathrm{q}, \mathrm{r}, \mathrm{P}_{\mathrm{x}}, \mathrm{P}_{\mathrm{y}}, \mathrm{H}\right]$. Theory of inverse dynamic can be used to construct a carrier landing control system. 


\section{Control System Design}

\subsection{Principle of dynamic inverse}

A nonlinear system can be described as formula 1 . In formula $1, \mathrm{u}$ is the input of the system, $f(x)$ and $g(x)$ are nonlinear continuous function, specially, $g(x)$ should be a square matrix, if $|g(x)| \neq 0$, $\mathrm{u}$ can be expressed as follows.

$u=g^{-1}(x)[\dot{x}-f(x)]$

Then replace $\mathrm{x}$ with desired rate of change $x_{\text {des }}$ to get control law as:

$u=g^{-1}(x)\left[\dot{x}_{d e s}-f(x)\right]$

Commonly, $x_{d e s}$ can be in direct proportion to the difference of our command to state variable and its actual value, namely,

$\dot{x}_{\text {des }}=P\left(x_{c m d}-x\right)$

\subsection{Control system constitution}

According to the response rate and degree of accurate, control system is divided into three loops.

The inner loop (angular rate loop) and outer loop (angle loop) can be designed through theory of utilizing inverse dynamics. It is worth mentioning that in outer loop, $\Phi, \theta, \beta$ are chosen as state variables and $p, q, r$ as input, which is not a precise description of system because firstly, the influence of manipulation surface on state variables $\Phi, \theta, \beta$ is ignored. Second, there is assumption that when outer loop begin to work, inner loop has already arrived steady which in fact is not exactly true [7]. To avoid possible instability brought by this modeling inaccuracy, a PID control is added in this loop (shown in fig 1).

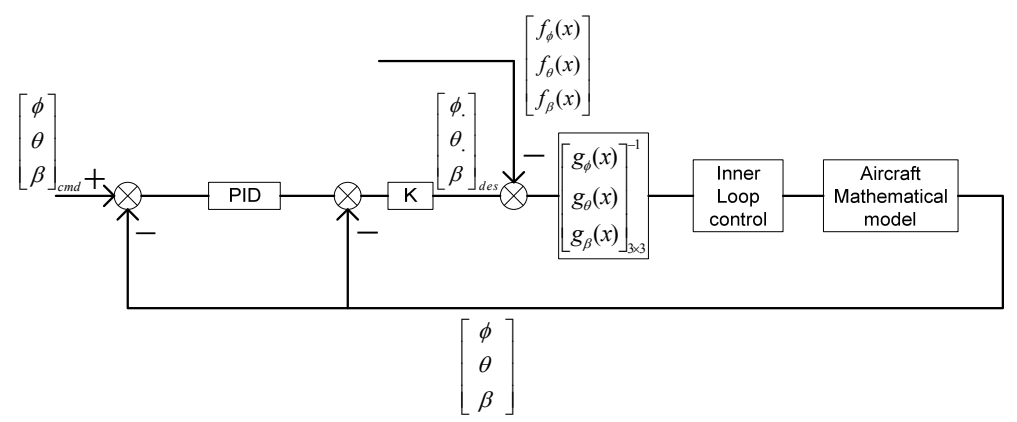

Fig. 1 Outer loop control design

As for path control loop, flying velocity $V$, lateral deviation $P_{x}$ and flying altitude $H$ are chosen as state variables. It is unrealistic to utilize inverse dynamics here because outer loop's state variables $\Phi, \theta, \beta$ cannot be written as input $\mathrm{u}$ as form of formula 1 in relevant equations. To control flying velocity, attack angle $a$ and flying velocity $V$ are used as feedback to generate the command of engine [6]. For another two state variables, lateral deviation $P_{x}$, lateral deviation rate $P_{x}$, flying altitude $H$, sink rate $H$ and yaw angle $\psi$ are used as feedback to generate command of $\varphi$ and $\theta$ (shown in fig 3 ), meanwhile, motion of deck should be a compensation for aircraft's command, because compare to other motions, deck's heave and sway is larger and can thus cause more inaccuracy in carrier landing, These two elements are supplemented in control system. A PID control is added to enhance robust of control system. Sketch of complete system is shown in fig 4. In manipulation surface model, transfer function and position limit are set [6]. And to simplify engine model, assume that thrust is proportional to throttle opening $\delta_{p}$ which ranges from 0 to 1 .

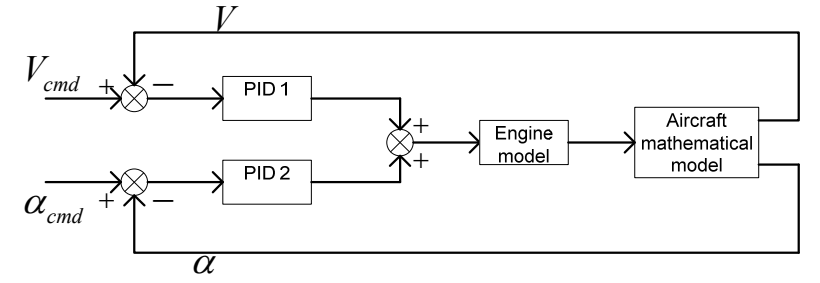

Fig. 2 Velocity control 


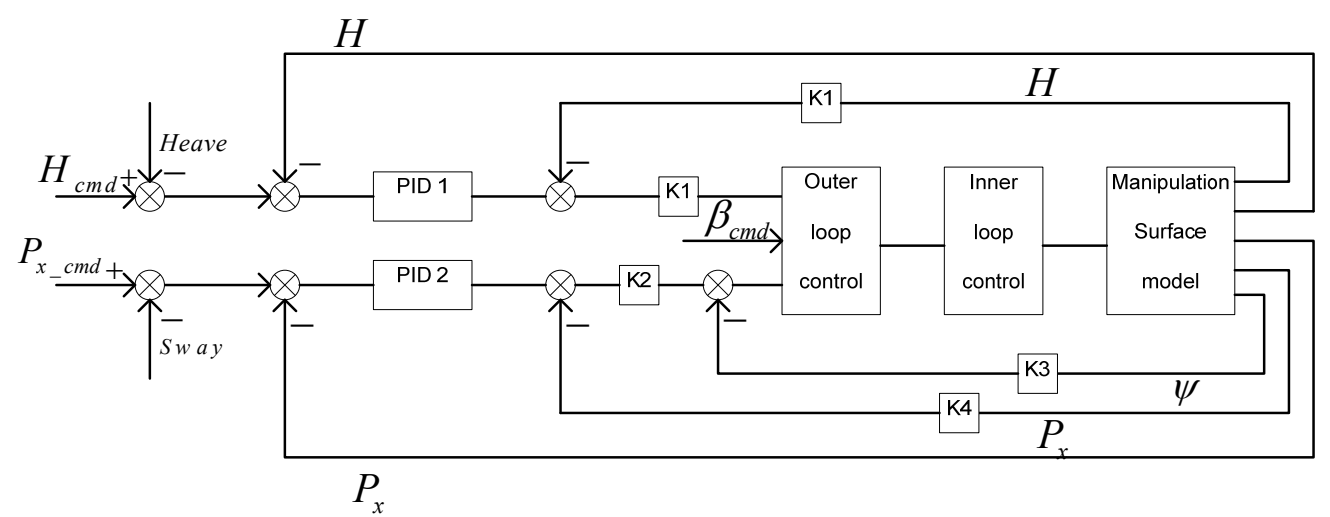

Fig. 3 Lateral deviation and height control

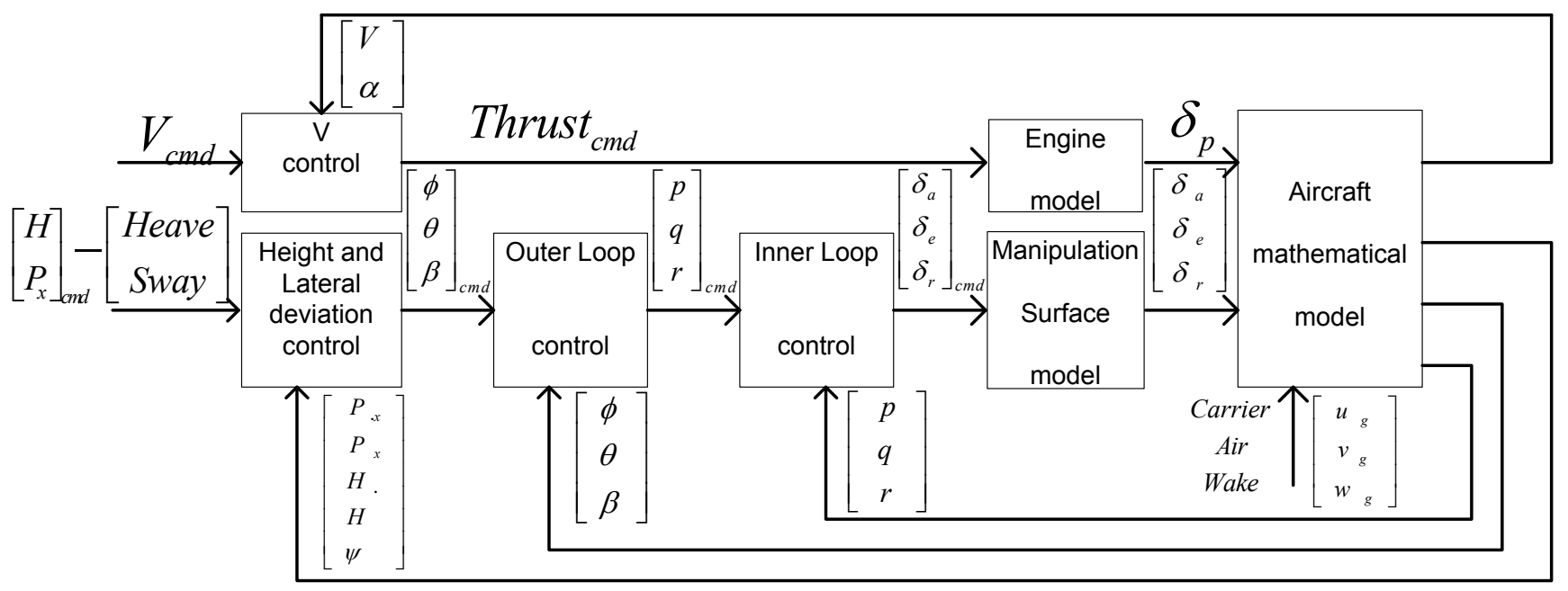

Fig. 4 Sketch of complete control system

\section{Simulation results}

Choose $F-18$ fighter as simulation aircraft. Simulation initial conditions are shown in table 2.

Table 2 Simulation initial conditions

\begin{tabular}{cc}
\hline Variables & Value \\
\hline $\mathrm{V}(\mathrm{m} / \mathrm{s})$ & 76.16 \\
$\alpha\left(^{\circ}\right)$ & 8 \\
$\beta\left(^{\circ}\right)$ & 1.22 \\
$\phi\left(^{\circ}\right)$ & 0 \\
$\theta\left(^{\circ}\right)$ & 4 \\
$\psi\left(^{\circ}\right)$ & 0 \\
$\mathrm{p}\left({ }^{\circ} / \mathrm{s}\right)$ & 0 \\
$\mathrm{q}\left({ }^{\circ} / \mathrm{s}\right)$ & 0 \\
$\mathrm{r}(\% / \mathrm{s})$ & 0 \\
$P_{x}(\mathrm{~m})$ & 0 \\
$P_{y}(\mathrm{~m})$ & -1316.8 \\
$\mathrm{H}(\mathrm{m})$ & 92.1 \\
Wind over the deck $(\mathrm{m} / \mathrm{s})$ & 15 \\
Carrier velocity $(\mathrm{m} / \mathrm{s})$ & 10 \\
Sea state level & 4 \\
Simulate Time $(\mathrm{s})$ & 20 \\
\hline
\end{tabular}

Construct control system shown and build carrier air wake model described above in Simulink. Path of centroid of aircraft in deck coordinate system is shown in fig 5-7, Aircraft's attitude angle curve is shown in fig 8-10. In order to compare, curves of simulating with control and without any 
control are both shown in one figure. Landing position error when exerting control is shown in table 3.

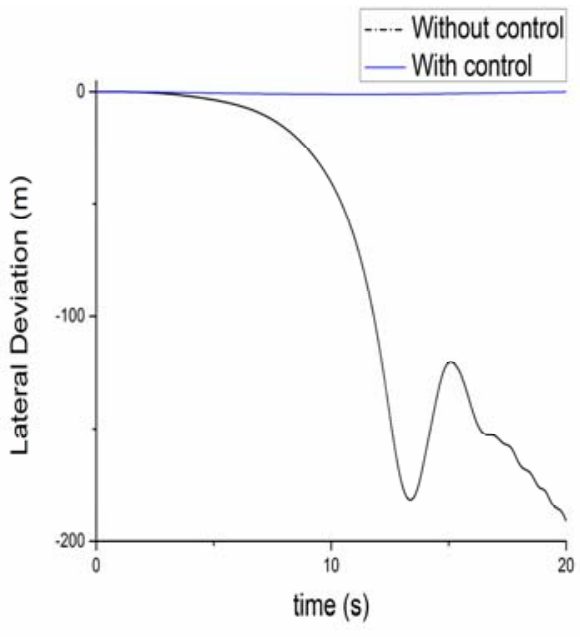

Fig. 5 Aircraft lateral motion

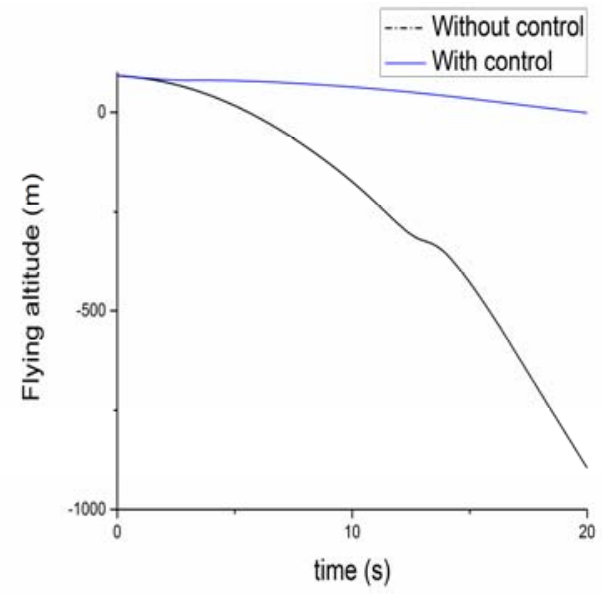

Fig. 7 Aircraft altitude

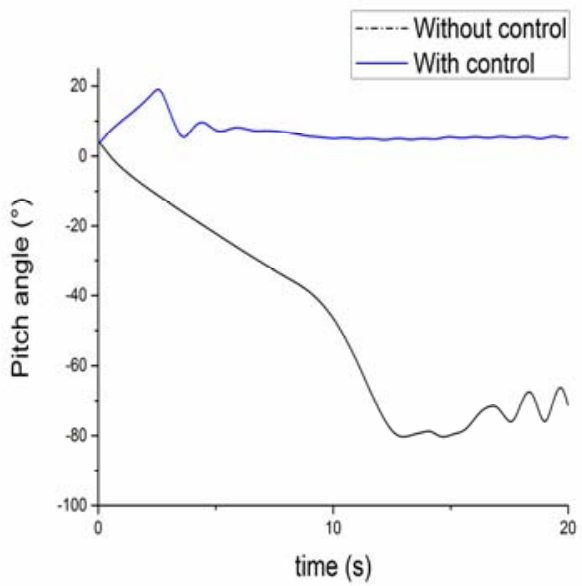

Fig. 9 Pitch angle

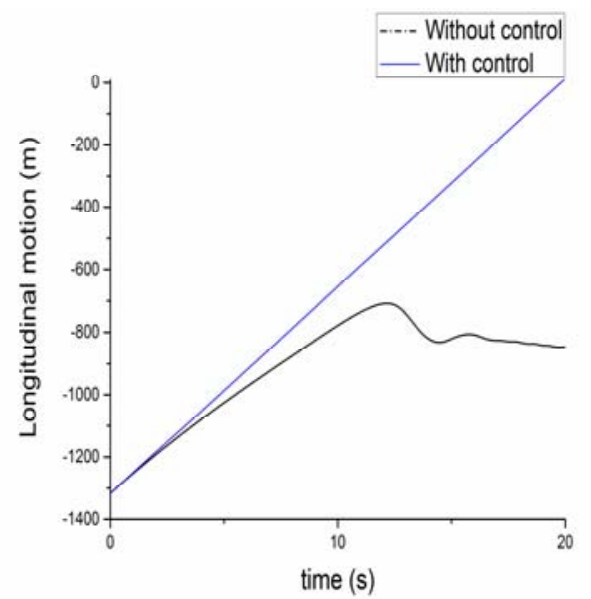

Fig. 6 Aircraft longitudinal motion

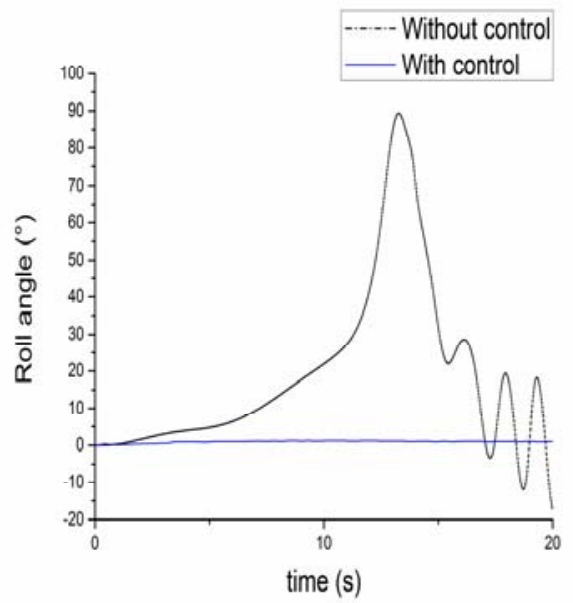

Fig. 8 Roll angle

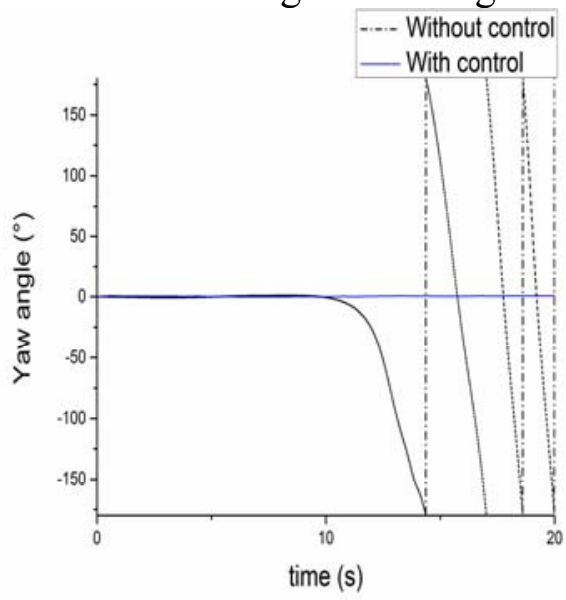

Fig. 10 Yaw angle

Table 3 Simulation carrier landing error

\begin{tabular}{cc}
\hline Error type & Value $(\mathrm{m})$ \\
\hline Longitudinal position & 0.94 \\
Vertical height & 0.068 \\
Lateral position & -0.092 \\
\hline
\end{tabular}

From table 3 , it is clear that through adding the control system described above, the lateral and height errors of touching point is in $1 \mathrm{~m}$ and longitudinal error is in $5 \mathrm{~m}$, indicatding that aircraft can land in ideal area. 
The attitude angle curve shows attitude angles will become close to their initial states after five seconds. Pitch angle of aircraft experienced a quick increase then decreased to normal range, this is because outer loop has a much slower response rate than inner loop does.

\section{Conclusion}

This paper present an auto carrier landing control system based on inverse dynamics and PID control. It is feasible and can effectively resist strong carrier air wake and make aircraft land in ideal area. This method has innovation significance and is worth further discussion and optimization.

\section{References}

[1]. Xu Dongsong, Liu xinyu, Wang lixin. Influence of changeful wind on landing safety of carrier based airplane. Journal of Beijing University of Aeronautics and Astronautics. Vol. 36 (2010) No. 1, p. 77-81.

[2]. SHI Liang. Influence of Disturbances on Ship-based Aircraft's Landing Process. Computer Simulation. Vol. 26 (2008) No. 12, p. 46-48,61.

[3]. GENG Jian-zhong, Yao Hai-lin, ZHANG Hong. Studies on Effects of Air Wake on Slope and Landing Property of Carrier Aircraft. Journal of System Simulaion. Vol. 21 (2009) No. 21, p. 5940-5943.

[4]. Zhang Weiguo, Li Aijun, Li Guangwen, Liu Xiaoxiong. Modern Flight Control System Design(in Chinese). Northwestern Polytechnic University Press, 2009, p. 1-31.

[5]. Xiao Shunda, et al. Automatic flight control system Volume two (in Chinese).National Defence Industry Press, 1982, p. 185-205.

[6]. Standard of Carrier-Based Aircraft Equivalent model and Carrier Landing Control (in Chinese). National Defence Industry Press, 2013, p. 18-25.

[7]. An Jun: Numerical Simulation of Aircraft Carrier Airwake and Preliminary Study on Control Law for Automatic Carrier Landing (Degree of Master of Engineering, Huazhong University of Science and Technology, China, 2012). p. 35-50

[8]. Li Yingtao: Design of approach power compensation and automatic throttle control of carrierbased aircraft (in Chinese).(Degree of Master of Engineering, University of Electronic Science and Technology of China, China,2007). p. 10-31

[9]. Duan Zhen: Research on Some Key Technologies of Flight Control System of UAV (Degree of Doctor of Science in Engineering, University of Chinese Academy of Science, China, 2014). p. 11-59.

[10]. Chen Jingzhi: Research on Guidance Law and Control Technology for Vehicle (Degree of Master of Engineering, Nanjing University of Aeronautics and Astronautics, china, 2007). p. $25-53$.

[11]. Information on https://en.wikipedia.org/wiki/McDonnell_Douglas_F/A-18_Hornet 